\title{
Botulinum Toxin Injection Into the Cricopharyngeal Muscle for Syringobulbia-induced Dysphagia: A Case Report
}

\author{
Kiyoko Murata ${ }^{\mathrm{a}}$, Ken Ikeda ${ }^{\mathrm{a}, \mathrm{b}}$, Yuji Kawase ${ }^{\mathrm{a}}$, \\ Osamu Kano ${ }^{a}$, Yasuo Iwasaki ${ }^{a}$
}

\begin{abstract}
We report a patient treated with botulinum toxin (BTX) for syringobulbia-induced cricopharyngeal dysphagia. A 54 year-old man with prior history of posttraumatic syringomyelia developed dysphagia suddenly. Bulbar function revealed palatal paresis and complete aglutition without lingual paresis. A videofluoroscopic swallowing study disclosed non-relaxation of the cricopharyngeal muscles (CPM). Follow-up magnetic resonance imaging displayed progression of syringobulbia and ascending syringomyelia. Symmetric slitlike medullary cavities could cause CPM dysfunction. A thoracic syringoperitoneal shunt had no therapeutic effects on dysphagia. He refused to undergo a syringocisternal shunt or cricopharyngeal myotomy. BTX (20 units) was injected into the left CPM under electromyographic guidance. Five days later, he was able to eat and drink gradually. Clinicoradiological profile of our patient indicated that the interruption of bilateral inhibitory projections from the solitary nuclei to the ambiguus nuclei could elicit uncontrollable CPM. BTX is beneficial and hopeful treatment for cricopharyngeal dysphasia due to progressive syringobulbia.
\end{abstract}

Keywords: Syringobulbia; Dysphasia; Botulinum toxin; Cricopharyngeal muscle; Videofluoroscopic study; Electromyography

Manuscript accepted for publication January 30, 2012

${ }^{a}$ Department of Neurology, Toho University Omori Medical Center, 6-11-1, Omorinishi, Otaku, Tokyo, 143-8541, Japan

${ }^{\mathrm{b}}$ Corresponding author: Ken Ikeda. Email: keni@med.toho-u.ac.jp

doi:10.4021/jnr81w

\section{Introduction}

Deglutition has been described as a series of four phases: oral preparatory, oral, pharyngeal and esophageal phase. Dysphagia is defined as swallowing impairment. This symptom frequently occurs in variable neurological disorders [17]. Dysphagia may complicate further clinical management and affect the quality of life or outcome in patients. Incomplete relaxation and defective opening of the upper esophageal sphincter (UES) could contribute to the pathogenesis of dysphagia. The cricopharyngeal muscle (CPM) is the major component of the UES between the hypopharynx and the esophagus.

Syringobulbia is a pathological cavity in the medulla oblongata. Dysphagia has been reported in $40 \%$ of patients with syringobulbia $[8,9]$. Surgical procedures are the most common treatment for dysphagia due to syringobulbia [9, 10]. However, little is known about botulinum toxin (BTX) treatment for CPM dysfunction in patients with syringobulbia. Here we report a patient with syringobulbia-induced dysphagia that was ameliorated by BTX injection into the CPM.

\section{Case Report}

A 54 year-old man noticed sudden onset of severe dyspagia. He had prior history of lumbar spine injury at the age of 34 years. Gait disturbance had persisted from the age of 51 years. At 2 years ago, he developed weakness and numbness in the left hand. He was diagnosed with posttraumatic syringomyelia at the cervical to lumbar cord levels. A thoracic syringoperitoneal shunt was performed for dysphagia, motor and sensory deficits in the four extremities. Dysphagia was not improved after the surgery. He was admitted to our department for evaluation and therapy of bulbar dysfunction. Neurological examination revealed moderate to severe degree of weakness and atrophy in the distal muscles of four extremities. Muscle stretch reflexes were decreased in the four limbs and there were no Babinski's sings. Superficial sensation was decreased mildly below the $\mathrm{C} 3$ cord level. Vi- 


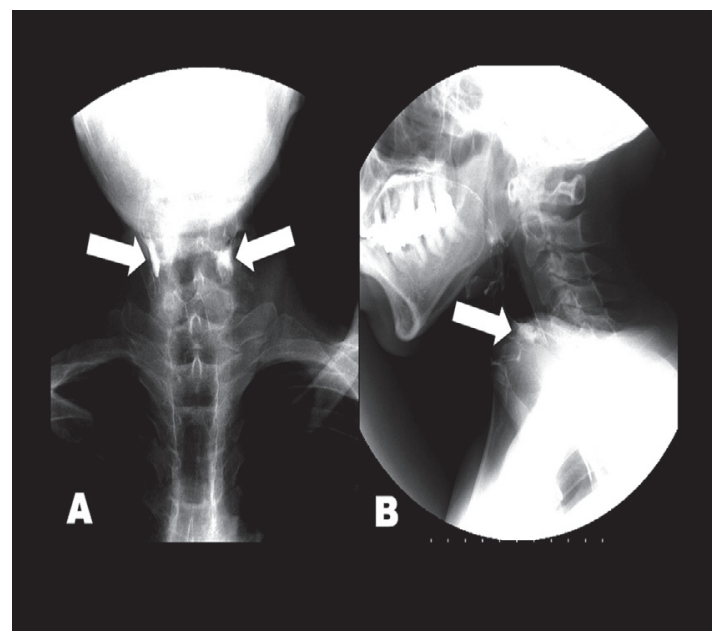

Figure 1. (A) Anteroposterior and (B) lateral views of esophagram. Barium retained at the level of esophageal entrance (arrows) and entered the trachea.

bration and proprioception sensation were not remarkable. Bulbar function showed complete aglutition and mild degree of palatal paresis. Hypoglossal nerve was normal.

A videofluoroscopic swallowing study disclosed non-relaxation of the CPM. Barium flowed into the trachea (Fig. 1).

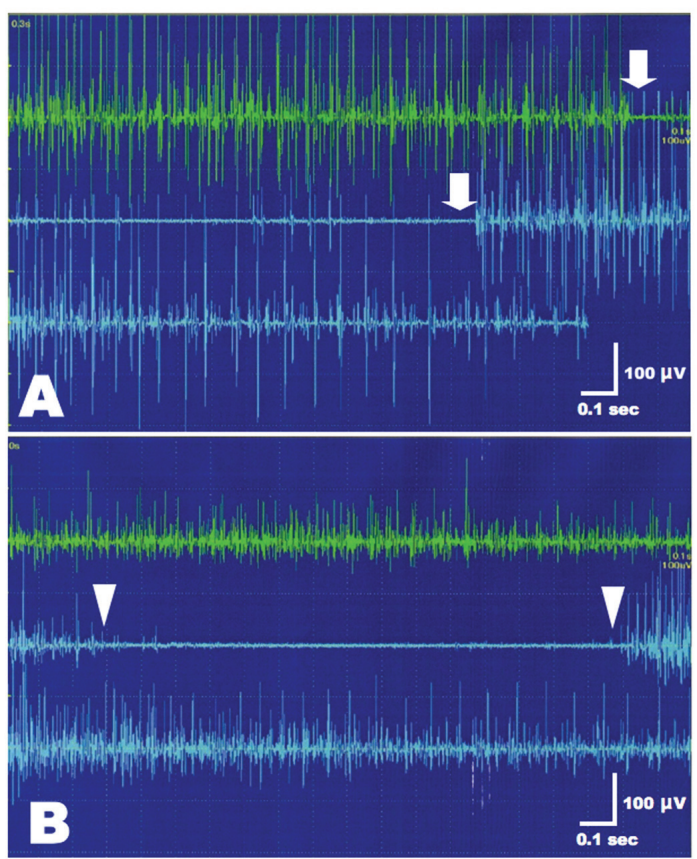

Figure 2. EMG of the left CPM before and after BTX treatment. (A) Incomplete inhibition of muscle contraction was present during the hypopharyngeal phase of swallowing (between arrows). (B) After BTX injection, CPM contraction amplitudes were decreased at rest compared to pre-treatment of BTX (upper green trace). Normal inhibition of CPM contraction was present during swallowing (between arrowheads).



Figure 3. Saggital views of spinal cord T2-weighted imaging. Widespread syringomyelia was found from the lumbar to high cervical cord.

For electromyography (EMG) record of the CPM, a coaxial needle-electrode was inserted through the skin at the level of cricoid cartilage, $1.5 \mathrm{~cm}$ posterior to its palpable lateral border, in the posteromedial direction. At rest, CPM generates tonic activity related to its function as a muscular sphincter (UES). Electrophysiological activities of the CPM disappear completely as the inhibitory pause during the hypopharyngeal phase of swallowing. Opening of the UES is defined as the EMG inhibitory pause of CPM when the amplitude of muscle contraction action was below $50 \mu \mathrm{V}$. EMG before BTX treatment revealed no inhibitory phase of CPM contraction during swallowing (Fig. 2A). Spinal cord magnetic resonance imaging (MRI) disclosed widespread syringomyelia (Fig. 3). Follow-up brain MRI displayed progression of syringobulbia. After dysphagia onset, symmetric slit-like medullary cavities were found (Fig. 4). These clinicoelectrophysiological and follow-up MRI studies supported the diagnosis of syringobulbia-induced CPM dysfunction.

He refused to undergo a syringocisternal shunt or cricopharyngeal myotomy. Informed consent of BTX injection was obtained from the patient and his family. Intramuscular injection of BTX was carried out under EMG monitoring of the CPM using a needle electrode-cannula with the characteristics of a monopolar electrode. This electrode was inserted in the neck at the level of cricoid cartilage as described above. Twenty units of BTX (Botox, GlaxoSmithKline, Japan), diluted 5.0 units $/ 0.1 \mathrm{~mL}$, were injected into the left CPM. Only the left CMP injection was performed because the right CPM was markedly shifted to the left side and the correct guidance was impossible. Bilateral injection had the risk of aspiration and vocal cord hypomotility in our patient. At 5 days after BTX treatment, the patient was able to drink and eat gradually without aspiration. EMG revealed reduction of muscle contraction amplitudes at rest and normal inhibition of CPM contraction during the hypopharyngeal phase of swallowing (Fig. 2B). The therapeutic effects of BTX persisted for 3 months without adverse events. Af- 


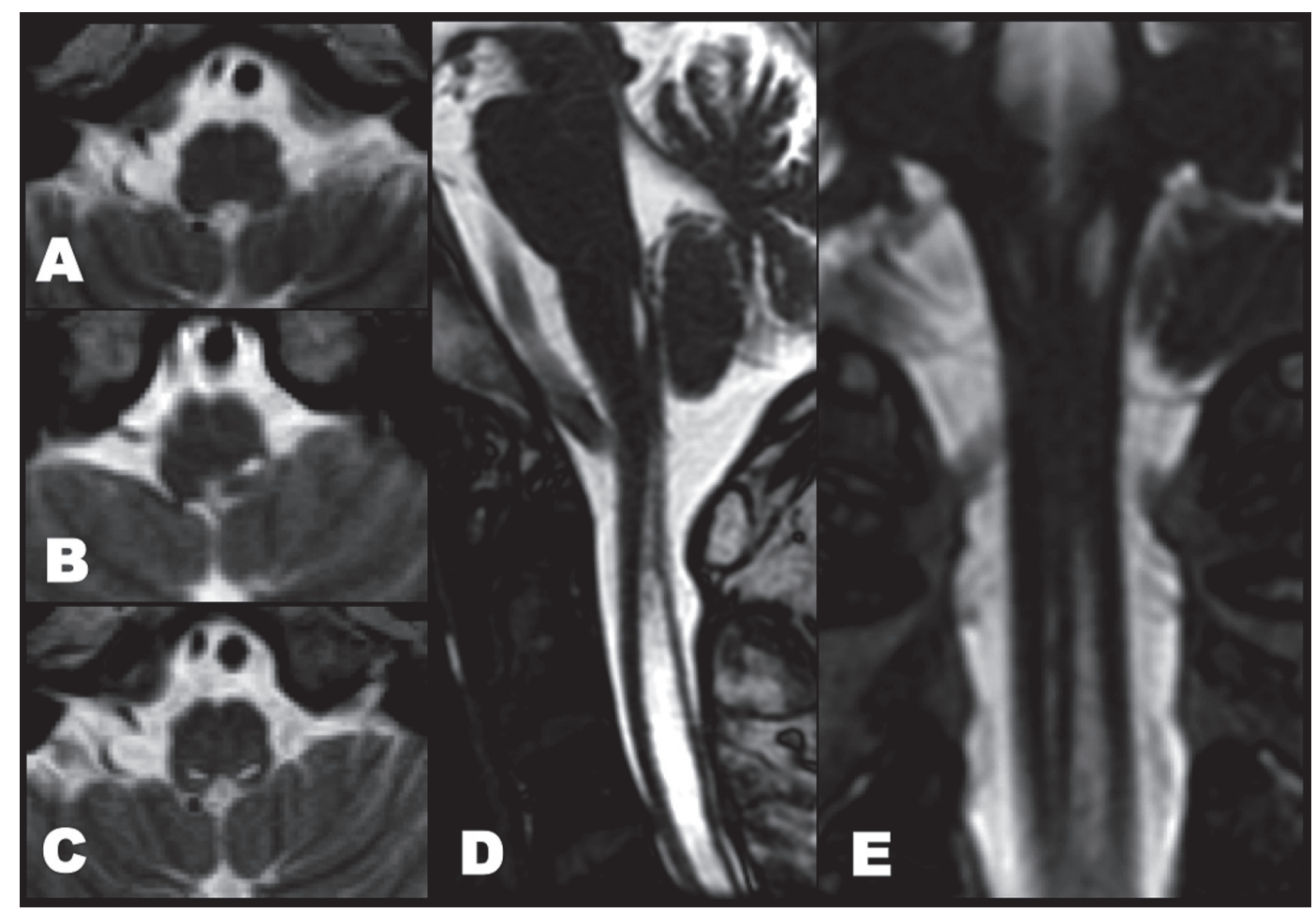

Figure 4. Follow-up T2-weighted imaging before and after onset of dysphagia. (A) No medullary syrinx at 2 years ago. (B) The left medullary syrinx was present at 6 months ago. (C) Axial, (D) sagittal and (E) coronal views of heavy T2-weighted spin-echo inverted imaging showed bilateral extension of medullary syrinx after bulbar onset.

terwards, 2nd BTX injection was performed.

\section{Discussion}

We described a patient with posttraumatic syringobulbiainduced dysphagia treated with BTX injection into the CPM.

Traumatic spinal cord injury may lead to the development of syringomyelia and the incidence ranges from $0.3 \%$ to $3.2 \%$ [11]. The pathophysiological mechanism of cavity formation might be implicated in vascular, hemorrhagic, or direct mechanical trauma from the original injury. Syrinx extension may occur by mechanical forces of the cerebrospinal fluid flow $[12,13]$. In the medulla oblongata, ascending syrinx usually spreads to an anterolateral direction from the floor of fourth ventricle [14]. Lower cranial nerve palsy is found frequently in patients with syringobulbia. Dysphagia due to CPM dysfunction was reported previously in a patent with syringobulbia [15]. Clinicoradiological progression of the present patient confirmed the concept that the interruption of bilateral inhibitory projections from the solitary nuclei to the ambiguus nuclei could play a crucial role in the pathogenesis of uncontrollable CPM.

The CPM acts as a muscular sling at the pharyngoesophageal junction and forms the UES. This muscle keeps a contracted state at rest and relaxes for a brief time dur- ing swallowing. Incoordination or hypertonus of the CPM may cause dysphagia and aspiration. CPM dysfunction has been identified in $5.7 \%$ of patients with neurologic disorder, $4.9 \%$ of patients with head and neck or esophageal tumors, and $8.9 \%$ of patients with other medical conditions [7]. In general, bougienage insertion or cricopharyngeal myotomy is a useful treatment for lowering the resting pressure of the CPM. However, the benefits of these procedures have been debated and the myotomy may trigger some complications $[16,17]$.

A videofluoroscopic swallowing study is performed recently for the diagnosis and clinical assessment of dysphagia. This method can evaluate the anatomy and physiology of the swallowing process. Moreover, electrophysiological investigation of oropharyngeal swallowing can detect CPM abnormalities during swallowing and can characterize the disturbance of CPM relaxation [6, 18]. Videofluoroscopic and EMG studies of swallowing function are valuable tests for the causal diagnosis and optimal therapy of dysphagia.

Otherwise, BTX has been used effectively for treatment of variable movement disorders, including blepharospasm, torticollis, writer's cramp, orofacial dystonia, spasmodic dystonia, and pharyngoesophageal segment spasm in tracheoesophageal puncture speakers [19-24]. This neurotoxin acts locally at the neuromuscular junction by binding to presynaptic cholinergic receptors and inhibiting the release of 
acetylcholine. Paralysis and virtually complete loss of miniature endplate potentials occurs after BTX injection into target muscles. Therapeutic effects usually start from 3 days after injection and persist on average of 3 months [19]. BTX injection of the UES has also been described for the treatment of dysphagia caused by hypertonus or defective relaxation of this muscle complex [22, 24, 25]. This treatment has been applied in patients with CPM dysfunction caused by various neurological disorders, including amyotrophic lateral sclerosis, multiple sclerosis, stroke and surgical injuries $[1,7,24,25]$. Schneider et al. [1] first mentioned BTX injection into the CPM for treatment of dysphagia. A recent electrophysiological study has reported that EMG monitoring during swallowing can predict responders of BTX injection into the unilateral CPM in patients with neurodegenerative diseases or stroke [6]. We first highlighted the safety and efficacy of BTX injection in a patient with syringobulbia associated cricopharyngeal dysphagia. Combined electrophysiological and videofluoroscopic swallowing examinations provided a critical determinant for CPM injection therapy of BTX in the present patient.

\section{Conclusion}

Clinicoradiological profile of our patient indicated that the interruption of bilateral inhibitory projections from the solitary nuclei to the ambiguus nuclei could cause uncontrollable CPM. BTX injection under EMG guidance is non-invasive and beneficial therapy for cricopharyngeal dysphasia due to progressive syringobulbia.

\section{Conflict of Interest}

All authors report no conflicts of interest.

\section{References}

1. Schneider I, Thumfart WF, Pototschnig C, Eckel HE. Treatment of dysfunction of the cricopharyngeal muscle with botulinum A toxin: introduction of a new, noninvasive method. Ann Otol Rhinol Laryngol. 1994;103(1):31-35.

2. Haapaniemi JJ, Laurikainen EA, Pulkkinen J, Marttila RJ. Botulinum toxin in the treatment of cricopharyngeal dysphagia. Dysphagia. 2001;16(3):171-175.

3. Ahsan SF, Meleca RJ, Dworkin JP. Botulinum toxin injection of the cricopharyngeus muscle for the treatment of dysphagia. Otolaryngol Head Neck Surg. 2000;122(5):691-695.

4. Blitzer A, Sulica L. Botulinum toxin: basic science and clinical uses in otolaryngology. Laryngoscope. 2001;111(2):218-226.
5. Alfonsi E, Versino M, Merlo IM, Pacchetti C, Martignoni E, Bertino G, Moglia A, et al. Electrophysiologic patterns of oral-pharyngeal swallowing in parkinsonian syndromes. Neurology. 2007;68(8):583-589.

6. Alfonsi E, Merlo IM, Ponzio M, Montomoli C, Tassorelli C, Biancardi C, Lozza A, et al. An electrophysiological approach to the diagnosis of neurogenic dysphagia: implications for botulinum toxin treatment. J Neurol Neurosurg Psychiatry. 2010;81(1):54-60.

7. Baredes S, Shah CS, Kaufman R. The frequency of cricopharyngeal dysfunction on videofluoroscopic swallowing studies in patients with dysphagia. Am J Otolaryngol. 1997;18(3):185-189.

8. Logue V, Edwards MR. Syringomyelia and its surgical treatment--an analysis of 75 patients. J Neurol Neurosurg Psychiatry. 1981;44(4):273-284.

9. Morgan D, Williams B. Syringobulbia: a surgical appraisal. J Neurol Neurosurg Psychiatry. 1992;55(12):11321141.

10. Bleck TP, Shannon KM. Disordered swallowing due to a syrinx: correction by shunting. Neurology. 1984;34(11):1497-1498.

11. Anton HA, Schweigel JF. Posttraumatic syringomyelia: the British Columbia experience. Spine (Phila Pa 1976). 1986;11(9):865-868.

12. Ball MJ, Dayan AD. Pathogenesis of syringomyelia. Lancet. 1972;2(7781):799-801.

13. Cho KH, Iwasaki Y, Imamura H, Hida K, Abe H. Experimental model of posttraumatic syringomyelia: the role of adhesive arachnoiditis in syrinx formation. J Neurosurg. 1994;80(1):133-139.

14. Larroche J-C. Malformations of the nervous system. In: Adams JH, Corsellis JAN, Duchen LW, eds. Greenfield's Neuropathology, 4th ed. New York: Edward Arnold; 1984: 385-450.

15. Fernandez F, Leno C, Combarros O, Berciano J. Cricopharyngeal dysfunction due to syringobulbia. Neurology. 1986;36(12):1623-1625.

16. Ellis FH, Jr., Crozier RE. Cervical esophageal dysphagia: indications for and results of cricopharyngeal myotomy. Ann Surg. 1981;194(3):279-289.

17. Ross ER, Green R, Auslander MO, Biller HF. Cricopharyngeal myotomy: management of cervical dysphagia. Otolaryngol Head Neck Surg. 1982;90(4):434-441.

18. Ertekin C, Pehlivan M, Aydogdu I, Ertas M, Uludag B, Celebi G, Colakoglu Z, et al. An electrophysiological investigation of deglutition in man. Muscle Nerve. 1995;18(10):1177-1186.

19. Jankovic J, Brin MF. Therapeutic uses of botulinum toxin. N Engl J Med. 1991;324(17):1186-1194.

20. Blitzer A, Komisar A, Baredes S, Brin MF, Stewart C. Voice failure after tracheoesophageal puncture: management with botulinum toxin. Otolaryngol Head Neck Surg. 1995;113(6):668-670. 
21. Crary MA, Glowasky AL. Using botulinum toxin A to improve speech and swallowing function following total laryngectomy. Arch Otolaryngol Head Neck Surg. 1996;122(7):760-763.

22. Dufour JF, Fawaz KA, Libby ED. Botulinum toxin injection for secondary achalasia with esophageal varices. Gastrointest Endosc. 1997;45(2):191-193.

23. Blitzer A, Brin MF. Laryngeal dystonia: a series with botulinum toxin therapy. Ann Otol Rhinol Laryngol. 1991;100(2):85-89.

24. DeVault KR. Dysphagia from esophageal diverticulosis responding to botulinum toxin injection. Am J Gastroenterol. 1997;92(5):895-897.

25. Blitzer A, Brin MF. Use of botulinum toxin for diagnosis and management of cricopharyngeal achalasia. Otolaryngol Head Neck Surg. 1997;116(3):328-330. 